\title{
The Endomembrane System: A Representation of the Extracellular Medium?
}

\author{
Mehmet Ozansoy • Yagmur Denizhan
}

Received: 30 January 2009 / Accepted: 6 May 2009 /

Published online: 13 October 2009

(C) Springer Science + Business Media B.V. 2009

\begin{abstract}
Both prokaryotic and eukaryotic cells share the basic mechanisms of secretory protein synthesis. However, unlike prokaryotes, eukaryotic cells posses a system of compartments, the so-called endomembrane system, which are involved in the synthesis process. A comparison of the prokaryotic and eukaryotic protein synthesis processes and particularly the observation of the functional and structural similarity between the prokaryotic cell membrane (the interface to the cell exterior) and the membrane of the eukaryotic endoplasmic reticulum (one of the compartments within the endomembrane system) inspire a description that refers to either the eukaryotic endoplasmic reticulum or its membrane or the endomembrane system altogether as a representation of the extracellular medium. However, unless the terms in such a statement are carefully analysed and refined the description would be just a colloquial usage of the concept of "representation" rather than a biosemiotic statement. Another problem associated with employing the concept of "representation" in a biosemiotic context is due to the fact that it may evoke the impression of a conscious mind as the "owner" of the representation. In this paper theories about the emergence of the eukaryotic endomembrane system, as well as its functionality within secretory protein synthesis will be analysed in order to specify in what sense the concept of "representation" can be employed in this context without implying consciousness. Such a study is expected not only to provide an insight into the conditions and assumptions under which this concept can be used for lower level biological processes but also to shed some light on how representations emerge in general.
\end{abstract}

Keywords Endomembrane system $\cdot$ Secretory protein synthesis $\cdot$ Representation · Evolution

M. Ozansoy

Department of Molecular Biology and Genetics, Neurodegeneration Research Laboratory, Bogazici University, Istanbul, Turkey

Y. Denizhan $(\bowtie)$

Electrical and Electronics Engineering Department, Bogazici University, Istanbul, Turkey e-mail: denizhan@boun.edu.tr 


\section{Introduction}

Secretory protein synthesis is a vital process in cellular functioning. Although most of the mechanisms within this process are the same in prokaryotic and eukaryotic cells, in the latter the final stages of the process involve the endomembrane system, a system of compartments specific to eukaryotes alone. In eukaryotes secretory proteins are synthesised towards the inside of the endoplasmic reticulum (ER), a membrane-bound compartment that is part of the endomembrane system, in a very similar way they are synthesised towards the cell exterior in prokaryotes. Particularly the membrane of the endoplasmic reticulum (one of the compartments within the endomembrane system) assumes at those stages a functionality very much resembling that of the cell membrane (the interface to the cell exterior) of prokaryotes. Employing a metaphorical description one could say that the eukaryotic cell produces, tries out and fits the proteins that are going to be secreted to the extracellular medium first on the endoplasmic reticulum.

The observation of this fact inspires an intuitive description that refers to either the eukaryotic endoplasmic reticulum or its membrane or the whole endomembrane system as a "representation" of the extracellular medium, in a way similar to saying that a mannequin used by a tailor for fitting dresses is a "representation" of the person who is going to wear the dress. No matter how obvious the usage of the concept of "representation" sounds in the realm of human activity (like in the case of the tailor-mannequin example), a similar usage in a biological context turns out to create a variety of problems. First of all, there is an apparent ambiguity from the semiotic point of view whether one should consider the eukaryotic endoplasmic reticulum or its membrane or the whole endomembrane system as a representation of the extracellular medium. Second, it is questionable whether one of these candidate structures should be considered as a representation of the extracellular medium or of something else. Next, it is not clear at all to whom such a representation would make sense. Finally, a statement of the kind " $\mathrm{X}$ is a representation of $\mathrm{Y}$ for $\mathrm{S}$ " evokes the impression of consciousness on behalf of the subject $\mathrm{S}$, a rather undesired implication in the context of lower level biological processes.

As can be seen, the employment of the concept of representation in biological processes is connected with various essential problems, and a description of that kind cannot go beyond colloquialism unless the terms in it are carefully analysed, the ambiguities removed and the conditions of correct usage identified.

On the other hand, "representation"-if it can be rescued from the load of prior debates around representationism and unintended implications ${ }^{1}$ - can lend itself to an extended usage as a biosemiotic term and provide valuable contribution to the understanding of semiotic processes. This will only be possible if the above mentioned problems can be solved and ambiguities removed, a task which asks not only for a careful consideration of the evolutionary origins of the endomembrane

\footnotetext{
${ }^{1}$ Encyclopedia Britannica gives the following explanation for representationism: "philosophical theory of knowledge based on the assertion that the mind perceives only mental images (representations) of material objects outside the mind, not the objects themselves. The validity of human knowledge is thus called into question because of the need to show that such images accurately correspond to the external objects. The doctrine, still current in certain philosophical circles, has roots in 17th-century Cartesianism, in the 18thcentury empiricism of John Locke and David Hume, and in the idealism of Immanuel Kant."
} 
system and its functionality within secretory protein synthesis, but also an analysis of semiotic statements like " $\mathrm{X}$ is a representation of $\mathrm{Y}$ for $\mathrm{S}$ ". The reasons why the concept of representation can easily be employed in the realm of human activity while it creates a series of problems in biological context need to be clarified.

\section{Endomembrane System and its Evolution}

Within evolutionary time a lineage of prokaryote-like organisms (proto-eukaryotes) has diverged from Bacteria and Archaea and eventually gave rise to eukaryotes (Cavalier-Smith 2002). Eukaryotic cells differ fundamentally from their prokaryotic counterparts by their possession of internal, membrane-bound compartments, which allow a better organisation of cellular functions. These membrane-bound compartments provide the coexistence of a diverse range of environments within a single cell, thus an enormous diversity of functions that can be carried out.

In eukaryotic cells the bounding membrane of such compartments-including the nucleus and the so-called endomembrane system-seem to be folded and differentiated extensions of the cell membrane. The term "endomembrane system" refers to a group of functionally related compartments. Beside some other components this system consists of the endoplasmic reticulum, Golgi apparatus, lysosomes and secretory vesicles (Fig. 1).

The endoplasmic reticulum (ER) is a tubular network spreading throughout the cytoplasm creating within the cell a subspace (the so-called ER lumen), which can comprise about $10 \%$ of the total cell volume and is topologically equivalent to the cell exterior. This subspace is used for the storage of certain ions, basically $\mathrm{Ca}^{+2}$. Furthermore, ER lumen serves as an isolated environment for the synthesis of certain lipids, as well as for the correct folding of proteins that will be secreted to the extracellular environment or mounted on the outer leaflet of the cell membrane.

The Golgi apparatus is a system of stacked sack-like structures, again creating a specific subspace. The Golgi apparatus is the site of modification of the proteins folded in the ER lumen. There is continuous trafficking between ER, the Golgi apparatus and the cell membrane via vesicles which transport the proteins and lipids.

Fig. 1 Vesicular trafficking between the plasma membrane, endoplasmic reticulum, Golgi apparatus and lysosomes

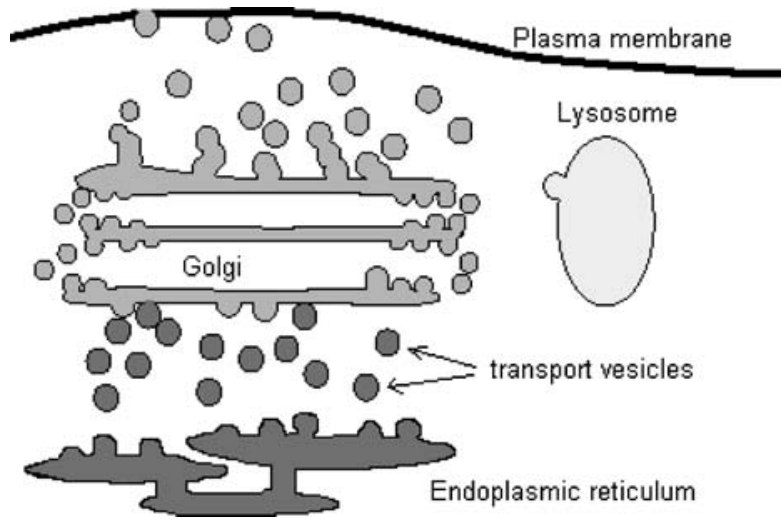


Some vesicles that bud off from the Golgi apparatus turn into lysosomes after their interior transforms into an acidic medium. Lysosomes are the site of degradation of extracellular materials which are engulfed by the cell; hence there is vesicular traffic between the cell membrane and lysosomes.

Comparative genomic and molecular evolutionary studies between lower and higher eukaryotic cells have revealed the following major results (Jekely 2007):

1 All eukaryotic cells including the least common eukaryotic ancestor have a fully equipped endomembrane system.

2 Even some prokaryotic organisms have precursors of a membrane trafficking system.

3 The evolution of proteins involved in endomembrane trafficking in different eukaryotic lineages exhibits an independent yet parallel increase in complexity.

4 Within the course of evolution of multicellular eukaryotic organisms the complexity of proteins involved in endomembrane trafficking increases even faster.

While the evolution of some organelles along the path from prokaryotes to eukaryotes is well-known, the present state of information about the evolution of the endomembrane system is relatively limited. Functionalities of the endomembrane system known so far include internalisation and digestion of extracellular materials, their targeted intracellular transport, as well as the surface remodelling of the plasma membrane and secretion of molecules into the extracellular environment.

So far two paradigms have been proposed for the evolution of the endomembrane system:

(a) Endosymbiotic origin: All endosymbiotic models posit that a prokaryote (or a precell) engulfs and integrates another prokaryote. Such a process is called nonphagotrophic internalisation as opposed to phagotrophic internalisation which refers to the engulfment and digestion of entire cells. The nonphagotrophic internalisation of a bacterium by another prokaryotic cell which is devoid of a dynamic cytoskeleton and endomembrane system is highly problematic. It is much more probable that phagotrophic cells that already have developed endomembrane dynamics acquire internal symbionts (the putative proto-nucleus, mitochondria and chloroplasts). Therefore every model of eukaryogenesis has to account for the origin of phagotrophy. However, none of the endosymbiotic models is sufficiently developed to explain why a prior endosymbiosis should have triggered the development of phagotrophy. If the order of origins is reversed, the problem disappears. Phagotrophy can easily account for the acquisition of symbionts (Margulis 1998).

(b) Autogenous origin: Autogenous models state that eukaryotic endomembranes evolved by the inward folding (invagination, tubulation or vesiculation) of the host cell's plasma membrane. Different autogenous models disagree about the nature and function of the first endomembranes but agree about the major steps of membrane topogenesis. All autogenous models have the following cell biological constraints:

- No intracellular compartment could have segregated before the origin of transport between the topologically segregated membranes, which allows balanced membrane growth and turnover. 
- As the secretory endomembrane system segregated topologically from the plasma membrane it had to contain the ribosome docking apparatus. The topological segregation and the redirection of a novel transport system for the proteins to be secreted or mounted on the extracellular sites of the cell membrane was a key event during the origin of eukaryotes.

The first detailed autogenous models proposed that the origin of nutrient uptake, either by endocytosis (engulfing of macromolecular structures) or phagocytosis (engulfing of entire cells) was the initial step in the evolution of the endomembrane system. Phagocytosis, the engulfment and digestion of entire cells, requires the coordination of at least three processes:

- Sensing of and binding to the prey

- Membrane remodelling around the prey

- Secretion of digestive enzymes and food uptake.

The question arises in what order these elementary steps evolved. Clearly membrane remodelling is useless if the prey is not digested and absorbed. On the contrary, prey binding, digestion and food uptake can happen - although not very efficiently - without the internalisation of prey. Such considerations led to the idea that the elaboration of a membranous secretory system was the first step in the origin of eukaryotic endomembranes (Jekely 2003, 2007; Margulis 1998; Saraste and Goud 2007; White and von Heijne 2004; Schnell and Hebert 2003; Glick 2002).

In spite of these controversies most cell-biologists accept some version of the autogenous scenario for the emergence of the endomembrane system (Jekely 2007). According to this view, the endomembrane system seems to have emerged possibly as a result of a failure in completing the digestion stage of an engulfment process in proto-eukaryotes. The residual internal structures of this failed process-if passed over many generations via cell division-may have evolved first into the endoplasmic reticulum then with the subsequent emergence of the Golgi apparatus into the complete functional compartment system called endomembrane system. Figure 2 shows a simplified graphical representation of this plausible evolutionary scenario over many generations. Although hard to provide experimental evidence, the topology of both ER and the Golgi apparatus in eukaryotes evokes the suspicion that its stacked and convoluted structure may have resulted from the topological

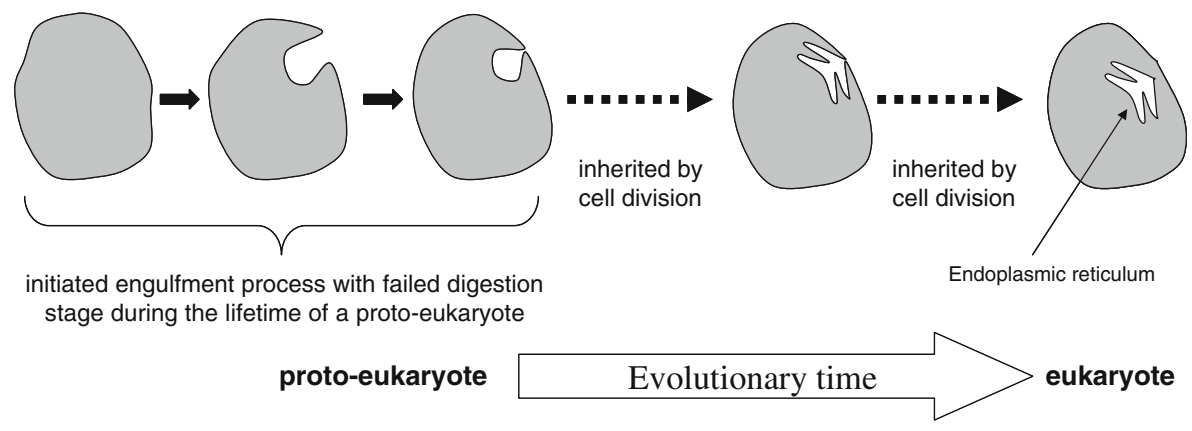

Fig. 2 A plausible scenario for the emergence of the endomembrane system as a consequence of an engulfment process with uncompleted digestion stage 
transformations throughout a long sequence of cell divisions. From the figure one can observe clearly that the inner sides of the ER and the Golgi apparatus are topologically equivalent to the outer side of the prokaryotic plasma membrane.

\section{Comparison of Secretory Protein Synthesis in Prokaryotes and Eukaryotes}

It is interesting to observe how the part of the prokaryotic secretory protein synthesis at the plasma membrane has been replaced by a more complex process involving both the endomembrane system and the plasma membrane in the eukaryotes. Table 1 provides a comparison of the secretory protein synthesis processes in prokaryotic and eukaryotic cells (Figs. 3 and 4).

As can be clearly observed from the 4th and 5th stages in Table 1, the equivalence of the eukaryotic ER to the cell exterior as seen by the prokaryote is not only topological but also functional as far as secretory protein synthesis is concerned. From the biological perspective this observation may not have much significance other than providing a support for the autogenous origin of the endomembrane system. But from the biosemiotic point of view a detailed analysis of this equivalence can provide a valuable clue about the nature of the sign relation at hand.

\section{Systemic and Semiotic Interpretations}

Without loss of generality one can say that during the course of evolution the eukaryotic ER membrane has taken over some part of the functionality of the prokaryotic plasma membrane, while the Golgi apparatus has introduced a novel functionality (of some posttranslational modifications and a final check for correct folding that do not exist in prokaryotes). A closer look at the evolutionary transition from the prokaryotic secretory protein synthesis to the eukaryotic one evokes the following considerations:

(i) The innovation in this transition is the addition of extra stages which allow "quality control" and correction of misfolded proteins. The complexity increase resulting from the integration of these additional stages, however, does not require a radical change in the whole process and does not disrupt functional continuity thanks to the modularity of the process. As a matter of fact the property of modularity is strongly related to the semiotic character ${ }^{2}$ of the considered process. If the eukaryotic ER membrane has partially taken over some of the functionalities of prokaryotic plasma membrane (and thus processes involving the endomembrane system have been inserted as an intermediate module into the overall process), it is thanks to the fact that only some aspects of the prokaryotic plasma membrane and of the cell exterior seen across it are

\footnotetext{
${ }^{2}$ The semiotic character resides in the property of involving only some but not all aspects of the object as stated by Peirce: "The sign stands for something, its object. It stands for that object, not in all respects, but in reference to a sort of idea, which I have sometimes called the ground of the representamen." (A Fragment, CP 2.228, c 1897)
} 
Table 1 Comparison of secretory protein synthesis in prokaryotes and eukaryotes

\begin{tabular}{lll}
\hline Stage \# $\quad$ Prokaryotic cell & Eukaryotic cell
\end{tabular}

1 DNA unwinds and mRNA is synthesised. (Fig. 3a)
Ribosomes bind to synthesised mRNA and translation starts. (Fig. 3b)

The translation process is arrested when the targeting signal (a certain amino acid sequence specific for secretory proteins) is produced and triggers the binding of the nearby Signal Recognition Particle (SRP) to the molecular complex consisting of mRNA, ribosomes and partially synthesised polypeptide chains

The molecular complex containing mRNA, ribosomes and partially synthesised polypeptide chains, is transported to the plasma membrane, binds to the specific SRP-receptor mounted on the plasma membrane, the SRP is released and translation resumes allowing the synthesis of the polypeptide chain through the translocon towards the cell exterior. (Fig. 3c)

The cleavage of the targeting signal by the signal peptidase (located on the outer leaflet of the plasma membrane) triggers the release and correct folding of synthesised polypeptide chain. (Fig. 3d)
DNA unwinds and mRNA is synthesised. (Fig. 4a) Synthesised mRNA leaves the nucleus through the nuclear pores and enters the cytoplasm. (Fig. 4b)

Ribosomes bind to synthesised mRNA and translation starts (Fig. 4c)

The translation process is arrested when the targeting signal (a certain amino acid sequence specific for secretory proteins) is produced and triggers the binding of the nearby Signal Recognition Particle (SRP) to the molecular complex consisting of mRNA, ribosomes and partially synthesised polypeptide chains

The molecular complex containing mRNA, ribosomes and partially synthesised polypeptide chains, is transported to the ER membrane, binds to the specific SRP-receptor mounted on the ER membrane, the SRP is released and translation resumes allowing the synthesis of the polypeptide chain through the translocon towards the ER interior. (Fig. 4d)

The cleavage of the targeting signal by the signal peptidase (located on the inner leaflet of ER membrane) triggers the release and correct folding of synthesised polypeptide chain. (Fig. 4e)

Vesicles transport folded proteins from the ER to the Golgi apparatus for further processing. (Fig. 4f) Inside the Golgi apparatus some posttranslational modifications occur and folding of the proteins is checked. If misfolded proteins are detected, they are sent back to ER for correction or degradation

Correctly folded and modified proteins are transported from the Golgi apparatus to the plasma membrane via transport vesicles for secretion. (Fig. 4g)

relevant in secretory protein synthesis. The structural and functional similarity of the interfaces (membranes) allows such an insertion without any significant change in the previous stages. This logic sheds some light on the importance of semiotic processes for evolution, which typically requires small innovations within largely conserved frameworks.

(ii) The "quality control" and correction facilities provided by the intermediate stages in eukaryotes (as opposed to the relatively "blind" release of synthesised 

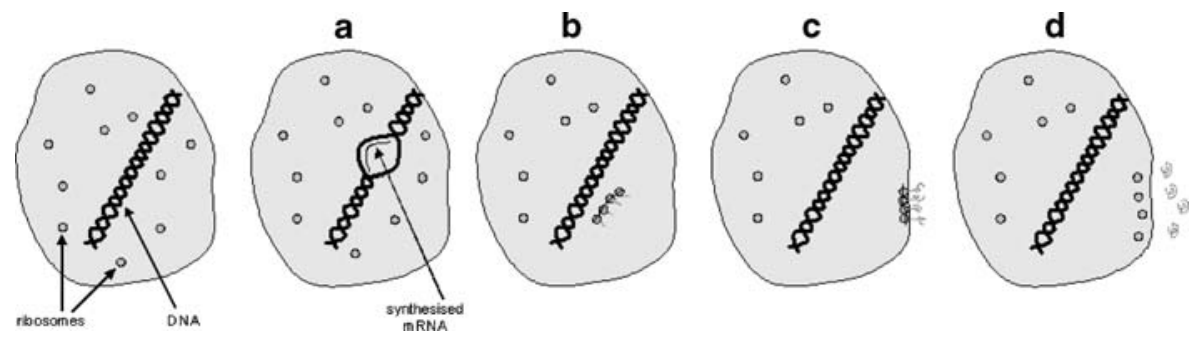

Fig. 3 Secretory protein synthesis in prokaryotes

proteins to the cell exterior in prokaryotes without a final check for correct folding) associate the "vicarious selection" theory proposed by Campbell (Campbell 1997). Campbell's Evolutionary Epistemology theory tells how in the course of evolution blind "trial" and elimination of the erroneous has been gradually replaced by a "knowledge-based" system that allows checking and improvement of the results of trials before they are actually performed. A problem associated with employing Campbell's Evolutionary Epistemology at a subcellular level is due to the fact that it may evoke the impression of some kind of "consciousness" on behalf of the organism that possess a "representation" of (or a model about) the "world". The emergence of eukaryotic endomembrane system constitutes a good example for a rather smooth transition from the relatively "blind release" of secretory proteins (i.e. without a final check for correct folding) to a process that involves "quality control" and the possibility of folding correction, without requiring any consciousness. This facility can be described as an intermediate unconscious mechanism that allows
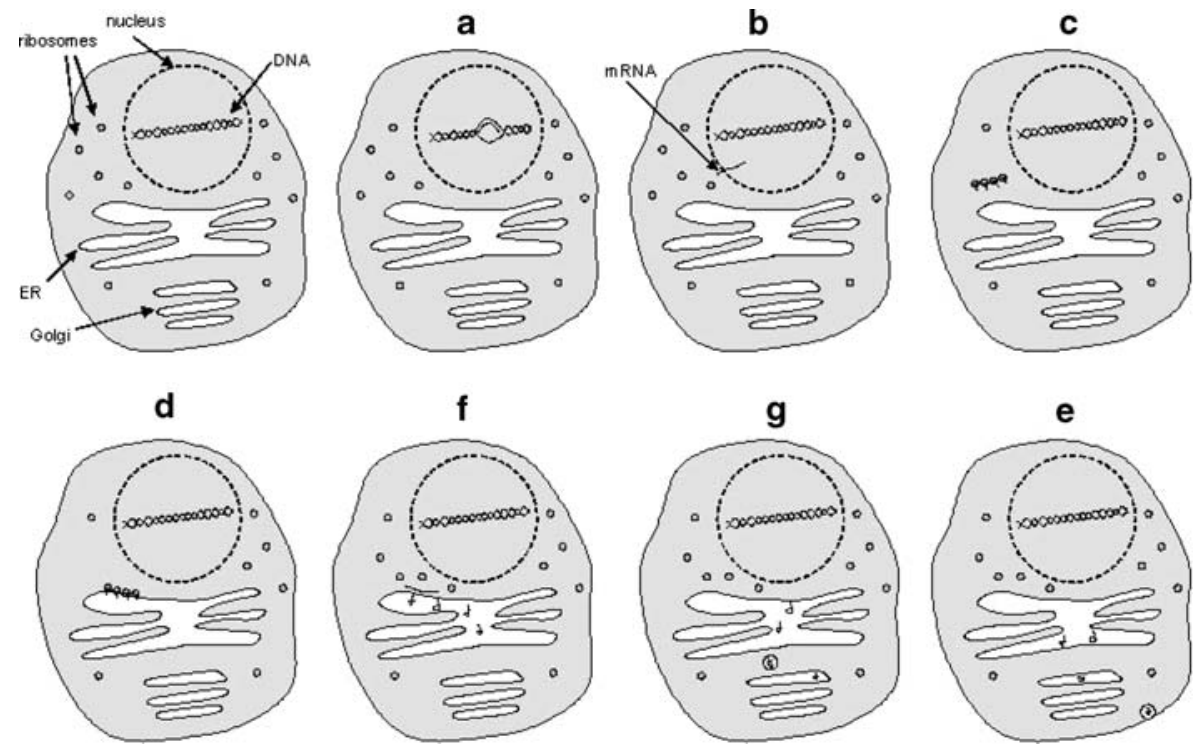

Fig. 4 Secretory protein synthesis in eukaryotes 
error checking and "thought experiments" on internalised models of the cell exterior. In that sense, the eukaryotic ER and its membrane can be considered as the relatively concrete part of the "representation" on which "trials" of secretory protein synthesis are made. On the other hand, the Golgi apparatus ought to harbour another (less known) part of this "representation" that serves as a reference in posttranslational modifications and particularly in error checking.

(iii) Independent of whether we are intending to refer to the ER, its membrane or the whole endomembrane system as a "representation" of something, a legitimate question is to whom this representation makes sense. Let us try to analyse a statement of the form " $\mathrm{X}$ is the representation of $\mathrm{Y}$ for $\mathrm{S}$ ": for $\mathrm{X}$ to be a representation of $\mathrm{Y}$ for $\mathrm{S}, \mathrm{S}$ must have "some prior acquaintance" with $\mathrm{Y}$. In an organismal context "prior acquaintance with something" can be translated as "a prior relation to something". Thus the emergence of a representation (X) can be regarded as the replacement of the relation $R(S \Rightarrow Y)$ by a relation $R(S \Rightarrow X)$. Following this logic, $\mathrm{R}(\mathrm{S} \Rightarrow \mathrm{Y})$ itself can be the result of a prior replacement. Therefore, rather than using $\mathrm{X}$ and $\mathrm{Y}$ it may be meaningful to introduce a formalism that bears explicit reference to the chronological order by using indices: $X=X_{n}, Y=X_{n-1}$. With this modified notation the emergence of a new representation $\left(X_{n}\right)$ for $S$ would be the last in a sequence of replacements: $R$ $\left(S \Rightarrow X_{n}\right)$ replacing $R\left(S \Rightarrow X_{n-1}\right)$ replacing $R\left(S \Rightarrow X_{n-2}\right)$ etc., that is to say, any new representation is a representation of an older one. At this point the logical problem of an infinite regress seems to arise if we cannot provide a grounding entity $X_{0}$. The relation $R\left(S \Rightarrow X_{o}\right)$ to such a grounding entity needs to be an absolute bottom line that cannot be further reduced to a prior relation. A solution to this riddle can be found in Gilbert Simondon's Theory of Individuation (Simondon 1992) that puts the origin of any individual into a preindividual state of ontic unity where the individual and its future environment exist only as a potential within an undifferentiated medium. As a matter of fact, Simondon states that there is an ongoing process of individuation rather than individuals. What we usually call individuals are snapshots of this process of individuation. Adopting this theory the primary relation $R\left(S \Rightarrow X_{o}\right)$ turns out to be that of an ontic unity where $S$ does not yet exist as an individuated entity but only as a potential within $\mathrm{X}_{\mathrm{o}}$, the preindividual state. Thus the very first replacement corresponds to the emergence of both $S$ and $X_{1}$ as separate entities out of $\mathrm{X}_{\mathrm{o}}$. According to Simondonian approach $\mathrm{S}$ should not be considered as an ultimate individual but an individuating entity that manifests itself in different forms at different stages of the process of individuation.

For the sake of simplicity let us apply this formalism first to the previously mentioned example of the tailor and the mannequin and try to identify the unstated assumptions that make the statement "for the tailor the mannequin used for fitting dresses is a representation of the person who is going to wear the dress" so obvious. Here $S$ is the tailor, $X_{n-1}$ the person who is going to wear the dress and $X_{n}$ the mannequin. Let us now try to identify the assumptions underlying this obvious description. The validity of the above statement depends on the unstated (but conventional) assumption that the tailor has prior experience of fitting dresses to 
persons directly. His relation to persons $\mathrm{R}\left(\mathrm{S} \Rightarrow \mathrm{X}_{\mathrm{n}-1}\right)$ has been replaced by a relation to mannequins $R\left(S \Rightarrow X_{n}\right)$. Without a reference to this prior stage in the evolution of the tailor's professional life the statement would not be valid. For example, for a tailor who has gained all his professional skills on mannequins alone, the statement would be meaningless. This example reveals the necessity of an evolutionary reference stage, no matter whether it is stated explicitly or assumed by convention. Another issue that needs to be addressed is the continuity of the tailor as he starts fitting dresses on mannequins rather than on real persons. Can the tailor at that later stage of his professional evolution still be considered as the same subject as before? Although he has changed in some sense again the convention dominates and we simply consider him as the same person. Such tacit conventions that remove many ambiguities are rather common in the domain of human activity, but not necessarily so in other fields, such that terms need to be carefully chosen in conformity with the formalism and explicitly stated.

Now we can try to apply the formalism to secretory protein synthesis: neither the subject $S$, nor the representations $X_{n}$ and $X_{n-1}$ are as obvious as in the previous case. Rather than that the choice is a matter of pragmatics, consistency and available evidence that can be used as a reference. In our case where we are concerned with making a statement about a representation in the eukaryotes, the eukaryotic cell seems the most obvious candidate for S. But such a choice would be problematic for the formalism which asks for the continuity of $S$ (analogous to the way the tailor is still considered as the same after changing his working conditions). In order to avoid this problem $\boldsymbol{S}$ can be chosen as a lineage of cells emerging from an undifferentiated primordial medium and extending through prokaryotes to eukaryotes. In accordance with the Simondonian concept, prokaryotes and eukaryotes can be regarded as snapshots of the process of individuation that governs the evolution along this lineage. The statement involving the concept of representation will be about the eukaryotic stage of $\mathrm{S}$. The next step has to be the choice of the evolutionary reference stage (analogous to the times when the tailor used to fit dresses on real persons). Here, available biological evidence imposes some conditions and offers the prokaryotic stage as a reasonable evolutionary stage of reference. In order to be able to make a very specific statement let us narrow down our scope to the secretory protein synthesis towards the inside of the endoplasmic reticulum (leaving the additional operations at the Golgi apparatus out of scope). The manifestation of the lineage $S$ at its prokaryotic reference stage (i.e. a prokaryotic cell) had some relation to the extracellular medium via its plasma membrane. Since the prokaryote has a direct relation only to its plasma membrane, it is reasonable to choose the prokaryotic plasma membrane as $X_{n-1}$. This choice also determines the next representation $X_{n}$ as the ER membrane of the eukaryote. Now the statement about the eukaryotic evolutionary stage of S can be made as follows: For the lineage (S) at its eukaryotic evolutionary stage, the ER membrane $\left(X_{n}\right)$ is a representation of the prokaryotic plasma membrane $\left(X_{n-1}\right)$ (Fig. 5).

If we want to trace down the sequence of representations further certain modifications will be necessary. In order to identify the prokaryotic plasma membrane $\left(\mathrm{X}_{\mathrm{n}-1}\right)$ itself as the representation of something else, first the evolutionary reference stage must be shifted backwards, yet there is not much evidence available about the evolutionary stages prior to prokaryotes. A possible hypothetical reference 


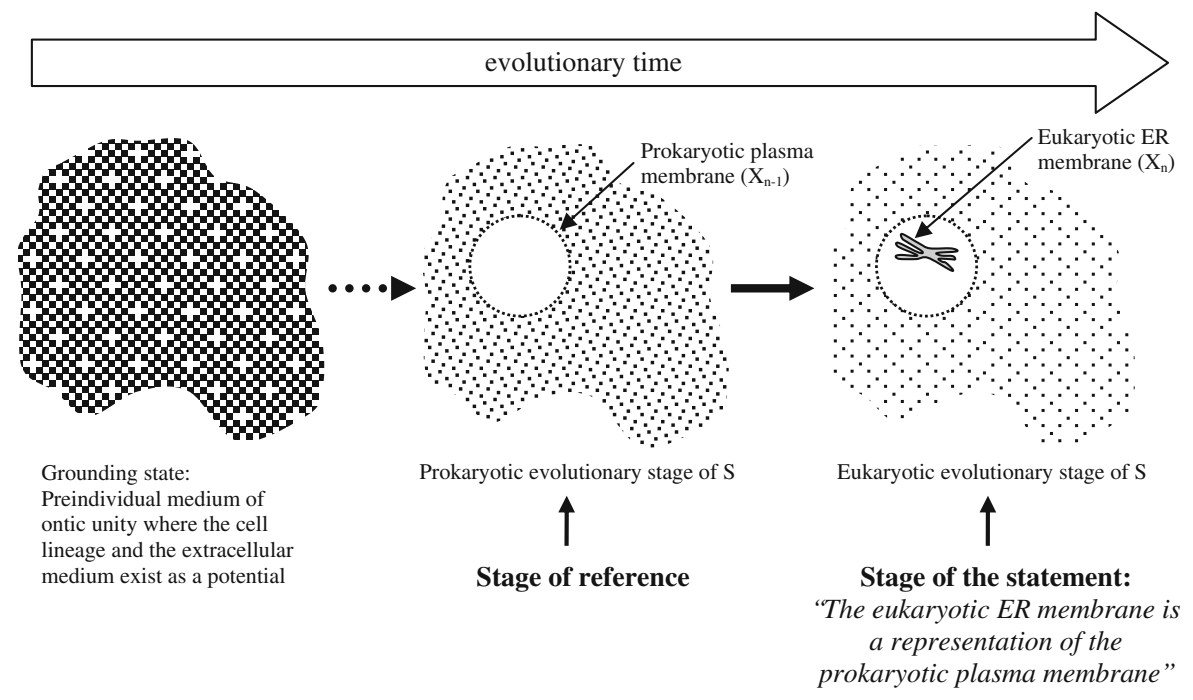

Fig. 5 Schematic explanation of the statement "The eukaryotic ER membrane is a representation of the prokaryotic plasma membrane for $S$ " where $S$ refers to the lineage emerging from the grounding state and extending through the prokaryote to the eukaryote

can, however, be made to the primordial medium out of which the ancestor of the prokaryote once emerged by developing a cell membrane. This primordial medium in which cells and their external environments resided only as a potential can be considered as the state of ontic unity $\left(\mathrm{X}_{\mathrm{o}}\right)$ and the evolutionary reference stage. For the most primal cell (the earliest manifestation of the lineage $\mathrm{S}$ ) the extracellular medium $\left(\mathrm{X}_{1}\right)$ would be a representation of the primordial medium $\left(\mathrm{X}_{\mathrm{o}}\right)$ out of which it is born. For $\mathrm{S}$ at all later evolutionary stages the plasma membrane $\left(\mathrm{X}_{2}\right)$ can be regarded as a representation of the extracellular medium $\left(\mathrm{X}_{1}\right)$ which, in turn, is a representation of the primordial medium $\left(\mathrm{X}_{\mathrm{o}}\right)$.

\section{Conclusion}

The modification in the secretory protein synthesis during the evolutionary transition from prokaryotes to eukaryotes constitutes an interesting case from a semiotic perspective because it inspires an intuitive description employing the concept of representation. However, the extension of this concept from the domain of human semiotics to lower biological processes is far from being straightforward and requires the identification of many assumptions that are conventionally taken for granted in human domain.

In order to handle this problem we have developed a formalism and applied it both to an example of a tailor fitting dresses on a mannequin and the eukaryotic secretory protein synthesis process in a comparative manner. According to this formalism the "owner" of the representation (analogous to the tailor) needs to be an evolving cell lineage rather than an individual cell while representations constitute a chain which starts with the "presence" of a primordial medium out of which the 
lineage is born and continues with its re-presentation, re-re-presentation etc., in other words every new representation is the representation of a former one within the continuum of evolutionary modifications. This approach that treats the evolution of representations as a process inseparable from biological evolution seems adequate for biosemiotic purposes and is accordant with Peircean semiotics at the same time. The idea of a chain of representations (each representation being the representation of a prior one) is compatible with Peirce's chain of signs, while the definition of the "owner" of representations (S) as a lineage accounts for the continuity of the "interpreter" which Peirce tacitly takes for granted in the chain. Indeed, in human semiotics the conservation of the interpreter along the chain of signs is acceptable (as is the conservation of the tailor in our example in spite of a change in the working conditions) but in a lower level biological process this continuity needs to be achieved by defining the "owner" of representations as an entity organised in time (the lineage).

There is another issue that needs to be clarified about the example of the tailor and the mannequin. In semiotic discussions when representations are mentioned (particularly in human semiotics) one usually means mental (internal) representations. However, the example we have given for the human domain involves an external representation (the mannequin) of the person who is going to wear the dress. This was a deliberate choice in order to avoid the controversies common to philosophical and semiotic discussions on representations and representationism which actually refer to mental representations. We do not want to address the issue of mental representations in the human context before developing a sound and acceptable formalism for the chain of emergences and the evolution of representations in living systems in general. Our formalism was actually an attempt in this direction trying to embed the emergence and evolution of representations into the ontogenetic process of individuation. Once the embodiment of the mind is established, one can start to consider mental representations as very late links in the chain of representations. By saying this we want to emphasise that we do not consider a conscious mind as a precondition and an inseparable companion of representations, rather than that we think that consciousness emerges at a later stage of this embodied evolutionary chain.

In the semiotic analysis of the eukaryotic secretory protein synthesis we have mainly concentrated on the part of the process at the ER membrane because it seems to constitute a rather concrete example of how a representation at the boundary (the representation of the extracellular medium in terms of the prokaryotic plasma membrane) has been internalised, turning into an "internal representation" in the literal sense. To put it in terms of the proposed formalism, the eukaryotic ER membrane can be regarded as a representation of the prokaryotic plasma membrane which re-presents the external medium.

The rest of the eukaryotic secretory protein synthesis process involving the Golgi apparatus has been left out of the scope of the semiotic analysis because (assuming the autogenous theory about the evolution of the endomembrane system to be correct) the Golgi apparatus is believed to have emerged later than ER and therefore seems to employ a later (thus much more complex, multifold internalised and distributed) form of representation of the extracellular environment, according to which posttranslational modifications and a final check for correct folding are made on synthesised proteins. 
The formalism developed as part of the efforts of making a statement about the eukaryotic secretory protein synthesis that involves the concept of representation seems to provide a framework suitable for biosemiotic and semiotic purposes in general. It is accordant or can easily be brought into accordance with Peircean semiotics while avoiding some of the problems that accompany representationism and implications about a conscious mind behind every kind of representation.

\section{References}

A Fragment, CP 2.228, c. 1897, http://www.helsinki.fi/science/commens/terms/representamen.html.

Campbell, D. T. (1997). From evolutionary epistemology via selection theory to a sociology of scientific validity. In: C. Heyes \& B. Frankel (Eds.), http://www.kli.ac.at/theorylab/Fulltext/campbell/campbell. html.

Cavalier-Smith, T. (2002). The phagotrophic origin of eukaryotes and phylogenetic classification of protozoa. International Journal of Systematic and Evolutionary Microbiology, 52, 297-354.

Glick, B. S. (2002). Can the Golgi form de novo? Nature Reviews Molecular Cell Biology, 3, 615-619.

Jekely, G. (2003). Small GTPases and the evolution of the eukaryotic cell. Bioessays, 25, 1129-1138.

Jekely, G. (2007). Eukaryotic membranes and cytoskeleton, origins and evolution Series: Advances in Experimental Medicine and Biology, 607.

Margulis, L. (1998). Symbiotic planet. New York: Basic Books.

Saraste, J., \& Goud, B. (2007). Functional symmetry of endomembranes. Molecular Biology of the Cell, $18,1430-1436$.

Schnell, D. J., \& Hebert, D. N. (2003). Protein translocons: multifunctional mediators of protein translocation across membranes. Cell, 112, 491-505.

Simondon, G. (1992). The genesis of the individual. In J. Crary \& S. Kwinter (Eds.), Incorporations (pp. 297-319). New York: Zone Books.

White, S. H., \& von Heijne, G. (2004). The machinery of membrane protein assembly. Current Opinion in Structural Biology, 14, 397-404. 